\title{
Pathophysiology of hemimasticatory spasm
}

\author{
G Cruccu, M Inghilleri, A Berardelli, G Pauletti, C Casali, P Coratti, G Frisardi, \\ P D Thompson, $M$ Manfredi
}

\begin{abstract}
Two patients aged 21 and 50 years presented with facial hemiatrophy and unilateral spasms of the masticatory muscles. Masticatory muscle biopsy showed normal findings in both patients and facial skin biospy specimens only showed atrophy, although morphoea (localised facial scleroderma) had been diagnosed nine years previously in the second patient. The involuntary movements consisted of brief twitches and prolonged contractions clinically and electromyographically similar to those of hemifacial spasm and cramps. The jaw jerk and the silent periods were absent in the affected muscles. Direct stimulation of the muscle nerve and transcranial stimulation of the trigeminal root demonstrated slowing of conduction and after-activity due to autoexcitation. Observations in other reported cases and these two patients suggest that hemimasticatory spasm is produced by ectopic activity secondary to focal demyelination of the trigeminal motor nerve fibres. The proposed cause of the neuropathy is focal damage to the masticatory nerves caused by compression, possibly resulting from the deep tissue changes that occur in facial hemiatrophy.
\end{abstract}

$(尹$ Neurol Neurosurg Psychiatry 1994;57:43-50)

Department of Neurosciences

G Cruccu

M Inghilleri

A Berardelli

G Pauletti

P Coratti

M Manfredi

Institute of Nervous and Mental Diseases C Casali

Department of

Odontology

G Frisardi

MRC Human

Movement and

Balance Unit and

University

Department of

Clinical Neurology,

Institute of Neurology, London, UK

P D Thompson

Correspondence to:

Dr Giorgio Cruccu,

Dipartimento Scienze

Neurologiche, Viale

Universita' 30, I-00185

Rome, Italy.

Received 12 January 1992

and in revised form

13 April 1993.

Accepted 19 April 1993

Hemimasticatory spasm (HMS) is a rare condition, characterised by unilateral forceful contractions of one or more masticatory muscles. In most patients it is associated with ipsilateral facial hemiatrophy. ${ }^{12}$ The cause of the spasms is not understood.

Kaufman, ${ }^{3}$ who first studied the electromyographic (EMG) characteristics of hemimasticatory spasm, stressed the close similarity with hemifacial spasm, and proposed that the spontaneous activity was generated in the trigeminal nerve fibres. Thompson and Carroll ${ }^{4}$ found similar EMG characteristics and also proposed a trigeminal neuropathy. Other authors, however, suggested CNS, sympathetic ganglia, or muscle dysfunction. ${ }^{56}$ Because none of the previously reported patients underwent masticatory nerve stimulation, conduction velocity and after-discharges could not be studied.

We studied two patients with HMS and facial hemiatrophy and demonstrated ectopic the trigeminal nerve. We also reviewed published reports of patients literature in whom a diagnosis of HMS was supported by EMG findings, to examine the presence of a common pathophysiological mechanism.

\section{Case reports}

PATIENT 1

A young man first noticed a hollowing of his left cheek at the age of 17 . One year later, brief involuntary twitches appeared in his left temporalis muscle. Clinical examination disclosed dilatation of the left pupil and the facial asymmetry; CT and magnetic resonance imaging (MRI) of the brain were normal. Left facial hemiatrophy was diagnosed.

At the age of 20 years, when the patient came to our observation, the involuntary movements were still restricted to the temporalis muscle. Small twiches alternated with severe and painful, prolonged contractions (lasting up to several minutes). Clinical examination showed only marked hypertrophy of the left temporalis muscle and hollowing of the cheek. Facial sensation was normal and the patient never complained of paraesthesias or neuralgia.

No clinical or laboratory evidence-including antinuclear antibodies, extractable nuclear antigen, native DNA, and mitochondrial antibody titres-was found of connective tissue disease.

The patient has been taking $1200 \mathrm{mg}$ carbamazepine daily for six months and reports moderate benefit. activity and slowing of motor conduction in

\section{PATIENT 2}

A 50-year-old woman first noticed small spots of abnormal pigmentation on the right side of the face at the age of 41 years. On her admission to a dermatological division, a skin biopsy was consistent with the diagnosis of localized scleroderma of the face, or morphoea. Corticosteroids were prescribed. At the age of 44 years she began to have involuntary twitches of the right masseter and temporalis muscles; the twitches increased in duration and frequency over the years.

At her first neurological assessment, the dyschromia was less apparent, but the face was frankly asymmetrical because of a slight hollowing of the right cheek and marked hypertrophy of the right masseter and temporalis muscles. The involuntary masticatory muscle contractions consisted of brief twitches and prolonged spasms, occurring many times a day either spontaneously, or 
more often, triggered by chewing, speaking or other voluntary movements of the mouth and jaw. Tonic contractions could last up to a few minutes; they were most painful and prevented the patient from opening her mouth. Like patient 1 , she had never had sensory s.rmptoms other than the pain induced by the muscle spasms. Neurological examination disclosed no abnormal signs apart from the spasms and hypertrophy of the masticatory muscles. MRI and CT scans showed hypertrophy of the right temporalis, lateral pterygoid, and masseter muscles, and dislocation of the right temporomandibular joint.

Serum tests for speckled and homogeneous antinuclear antibodies, and smooth muscle antibodies gave positive results; circulating immune complexes were increased. Extractable nuclear antigen, native DNA, and mitochondrial antibody titres were negative. No evidence was found of kidney, intestinal, or other systemic manifestations of vasculitis.

The painful spasms were attenuated by carbamazepine $600 \mathrm{mg}$ daily and transiently blocked by injection of local anaesthetics into the masseter and temporalis muscles. Diazepam was ineffective. After completion of all the investigations, injection of botulinum A toxin (30-50 U, Oculinum) into the affected muscles was repeated three times, with clinical benefit.

The following investigations were approved by the local ethical committee and both patients gave their informed consent.

\section{Investigations}

MUSCLE AND SKIN BIOPSY

In both patients, an elliptical biopsy specimen, including skin and underlying temporalis muscle, was obtained from the affected side. In patient 2 , a further skin biopsy specimen was taken from an area of slight dyschromia on the right forehead.

In both patients, the muscle was histologically normal and skin specimens only showed a flattened epidermis and atrophy of dermal appendages. In particular, no evidence of scleroderma was found in patient 2 , in whom morphoea had been diagnosed nine years beforehand.

A masticatory nerve biopsy was excluded because of the consequent functional damage.

\section{STANDARD EMG EXAMINATION}

The masseter, temporalis, suprahyoid, and facial muscles were bilaterally examined by concentric needle recordings (filters $50-5000$ $\mathrm{Hz}$ ). No abnormalities were found in either patient in the muscles clinically unaffected by the spasm, neither were denervation potentials found in the affected muscles. The voluntary and involuntary motor unit potentials examined were all normal, although difficulty in providing a steady level of voluntary activity and the frequent occurrence of involuntary activity hindered an exhaustive analysis of single motor unit potentials.

Paroxysmal spontaneous activity was recorded from the left temporalis in patient 1

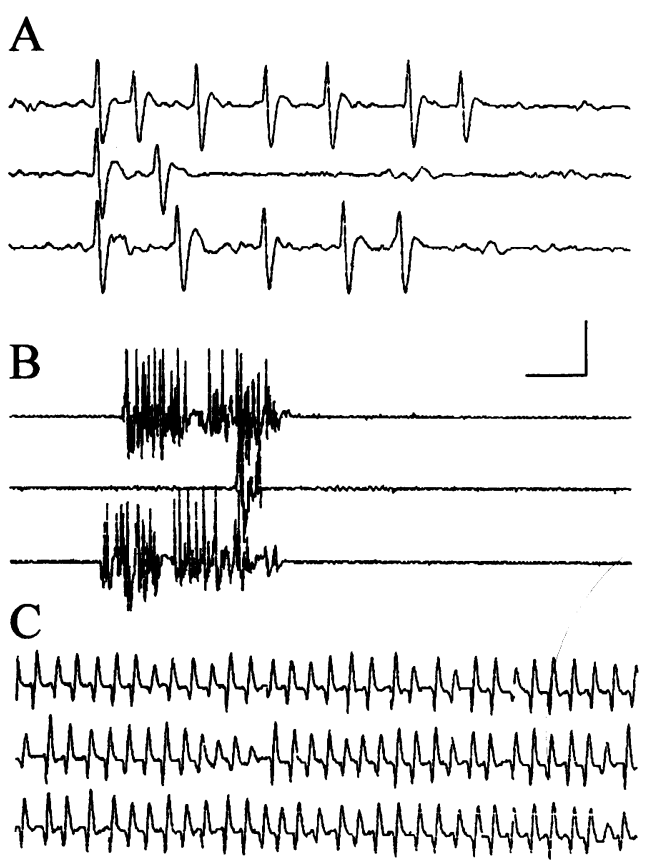

Figure 1 Involuntary EMG activity in patient 1. (A) High frequency discharges of a single motor unit potential. Calibration $10 \mathrm{~ms} / 1 \mathrm{mV}$. (B) Brief bursts of multiple motor unit potentials (corresponding clinically to twitches). Calibration $100 \mathrm{~ms} / 1 \mathrm{mV}$. (C) Large compound potential of multiple synchronised motor units discharging tonically at a $60 \mathrm{~Hz}$ frequency (corresponding clinically to prolonged spasms). Calibration $50 \mathrm{~ms} / 10 \mathrm{mV}$.

and the right masseter and temporalis muscles in patient 2. Involuntary EMG activity was similar in the two patients. Brief trains $(30-100 \mathrm{~ms})$ of two to seven single motor unit potentials reaching a discharge frequency of $100 \mathrm{~Hz}$ (fig $1 \mathrm{~A}$ ), or brief bursts (50-200 $\mathrm{ms}$ ) of multiple motor unit potentials occurred spontaneously, irregularly, and arrhythmically (fig 1B). The bursts would occasionally become more frequent and intense, with the progressive recruitment and synchronisation of more and more motor units, leading to a large $(12-15 \mathrm{mV})$ compound potential, discharging tonically at a high frequency (fig $2 \mathrm{C}$ ). The discharge frequency was always around $60 \mathrm{~Hz}$ in patient 1 and $70 \mathrm{~Hz}$ in patient 2 . This activity corresponded to the painful tonic contractions. The maximum duration of the contractions was 2 minutes in patient 1 and $30 \mathrm{~s}$ in patient 2 . In patient 2 , the prolonged spasm sometimes affected the temporalis muscle alone with complete electrical silence in the masseter or vice versa. At other times the spasm moved from one muscle to the other (fig 2), but never spread to involve the facial muscles or contralateral muscles of the jaw. In both patients, these patterns of involuntary EMG activity usually followed a strong voluntary clenching of the teeth, but could also be elicited by electrical stimulation of the mentalis or infraorbital nerves, though not the supraorbital nerve.

STIMULATION OF TRIGEMINAL MOTOR NERVE FIBRES

Signals were recorded through surface electrodes (filters $10-2000 \mathrm{~Hz}$ ) placed bilaterally 
Figure 2 Temporalis and masseter spasm in patient 2. Simultaneous recordings from right temporalis $(T)$ Large compound potentials of synchronised motor units discharging tonically in the temporalis muscle whereas the masseter is silent (1). After a few seconds, the number of motor units discharging synchronously in the temporalis decreases whereas similar activity starts in the masseter (2). The spasm ceases simultaneously in the two muscles (3). Calibration $50 \mathrm{~ms} / 2 \mathrm{mV}$. and masseter (M) muscles.
1

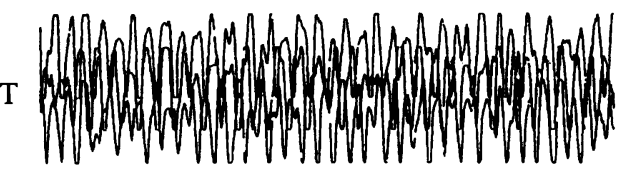

$\mathrm{M}$
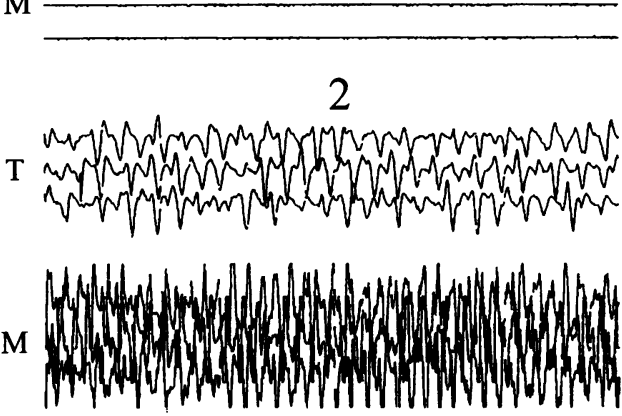

3

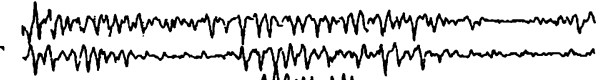

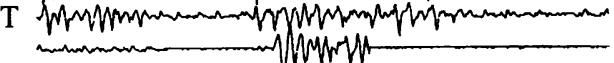

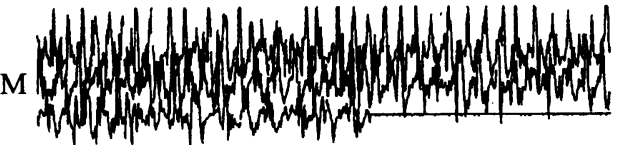

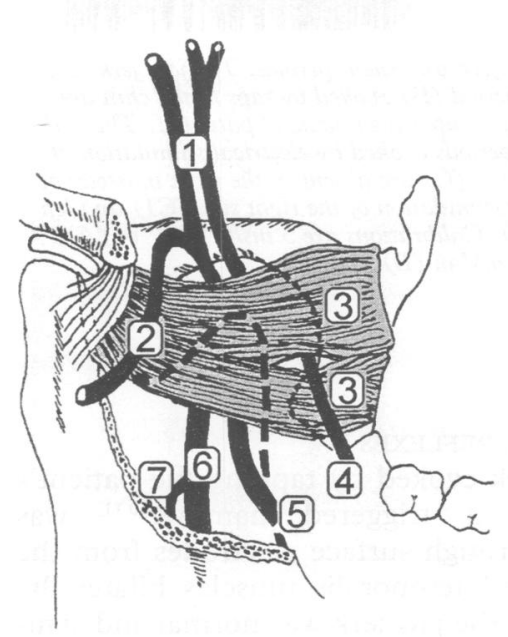

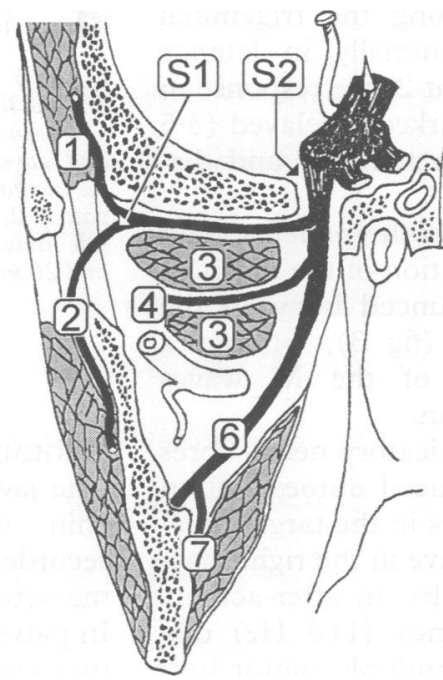

Figure 3 Schematic drawing of the infratemporal fossa and course of the masticatory nerves. Lateral view (left) and frontal section (right). Thi mandibular nerve leaves the cranial cavity through the foramen ovale of the greater wing of the sphenoid, and runs down into the infratemporal fossa, behind the lateral pterygoid muscle. At its exit from the foramen ovale it first gives off the motor branches that supply the jaw closing muscles. The temporo masseteric nerve turns laterally, running horizontally between the greater wing of the sphenoid and the upper belly of the lateral pterygoid muscle (3), and divides into the posterior temporal nerve (1) and masseteric nerve (2). The temporal muscle is also innervated by the median and anterior temporal nerves, which originate from the mandibular nerve and the buccal nerve (4). The deep temporal nerves (1) turn sharply around the temporal crest and run upwards between the skull and the temporal muscle. The masseteric nerve (2) turns downwards, passing between the zygomatic arch and the mandibular notch to reach the masseter muscle from its inner surface. The lateral pterygoid muscle is innervated by nerve branches (not shown) originating, close to the muscle, from the mandibular nerve and the buccal nerve; the medial pterygoid muscle is innervated by a small nerve (not shown), which originates slightly below the foramen ovale and remains posterior to the mandibular nerve. All the nerve branches that supply the jaw closing muscles originate before the mandibular nerve divides into its terminal mixed nerves: the lingual (5) and inferior dental (6) nerves. The jaw opening muscles are innervated by the mylohyoid nerve (7), which originates from the inferior dental nerve immediately before it enters the mandibular canal. The dashed line (left) represents the coronoid process of the mandible. $S 1$ and $S 2$ (right) indicate the approximate sites of nerve excitation with percutaneous needle stimulation (S1) and transcranial stimulation (S2). over the masseter and temporalis ${ }^{78}$ and by concentric needles inserted into the anterior temporalis.

The masseteric and deep temporal nerves in the infratemporal fossa (fig 3) were stimulated by delivering electrical shocks $(0.5-5$ $\mathrm{mA}, 0 \cdot 1 \mathrm{~ms})$ through two fine needle electrodes inserted below the zygomatic arch and anterior to the temperomandibular joint, and direct motor responses ( $M$ waves) were recorded from the masseter and temporalis muscle. ${ }^{78}$ In patient 1 , the masseter $M$ wave was normal and symmetrical; the left temporalis $M$ wave was slightly delayed (right latency $1.8 \mathrm{~ms}$, left latency $2.2 \mathrm{~ms}$ ) and normal in amplitude $(4.8 \mathrm{mV}$ on the right, $5 \cdot 1$ $\mathrm{mV}$ on the left). In patient 2 , the response in the right masseter was clearly delayed $(2.4 \mathrm{~ms}$ on the right, $1.5 \mathrm{~ms}$ on the left), although symmetrical in amplitude (6 $\mathrm{mV}$ on both sides) (fig 4).

The trigeminal root was stimulated transcranially ${ }^{10}$ by means of a high tension, low impedance electrical cortical stimulator (Digitimer), with the anode placed at the vertex and the cathode about $10 \mathrm{~cm}$ laterally, slightly anterior and superior to the ear. The

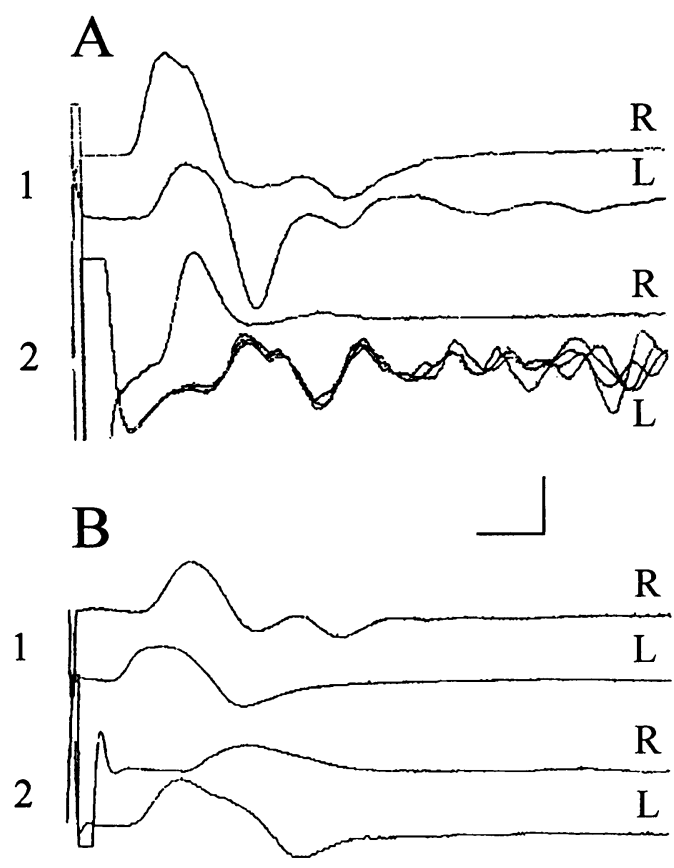

Figure 4 Motor conduction study. Recording from the right $(R)$ and left $(L)$ temporalis muscle in patient $1(A)$ and the right and left masseter muscle in patient $2(B)$.

Electrical stimulation of the masticatory nerves (masseteric and deep temporal nerves) in the infratemporal fossa (1) and transcranial stimulation of the trigeminal motor root

(2). In $(A)$, the direct motor responses evoked by stimulation of the deep temporal nerves (1) are only slightly asymmetrical, whereas those evoked by stimulation of the trigeminal motor root (2) are markedly delayed in the left temporalis. In (B), the responses evoked by stimulation of both the masseteric nerve (1) and the trigeminal motor root (2) are clearly delayed on the right masseter. In both patients, the amplitude of the responses to distal stimulations (1) is normal. Averages of four trials, except the bottom records in $(A)$, where four single trials are superimposed to differentiate the direct motor response (reproducible, time locked waves) from the late

asynchronous activity. Horizontal calibration is $2 \mathrm{~ms}$. Vertical calibration is $2 \mathrm{mV}$ in $(A)$ (except in the bottom records, where the calibration is $0.5 \mathrm{mV}$ ), and $5 \mathrm{mV}$ in (B). 
Figure 5 After-discharges evoked by stimulation of the masticatory nerves. (A) In the right masseter of patient 2 , the $M$ wave is followed by after-activity consisting of high frequency discharges of synchronised multiple motor unit

potentials lasting $250 \mathrm{~ms}$.

(B) In the left temporalis

of patient 1 , the $M$ wave is followed by late activity consisting of asynchronous motor unit potentials, starting after a brief pause of electrical silence. Calibration is $50 \mathrm{~ms} / 1 \mathrm{mV}$ in (A1), $10 \mathrm{~ms} / 2 \mathrm{mV}$ in (A2), and $5 \mathrm{~ms} / 1 \mathrm{mV}$ in (B)
A
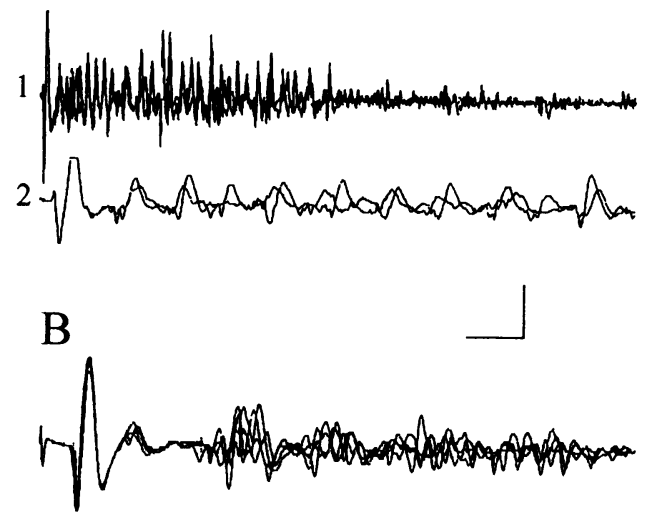

electrical field is thought to excite the trigeminal motor nerve fibres intracranially, near their exit from the skull ${ }^{10}$ (fig 3). In patient 1 , the responses from the masseter were normal and symmetrical. Because a large stimulus artifact and signals from facial muscles obscured surface recordings, responses from the temporalis muscle could only be examined by needle recording: in this condition, the latency of the response in the left temporalis ( $4 \mathrm{~ms}$ ) was markedly longer than that in the right temporalis $(2.5 \mathrm{~ms})$ and longer than the response simultaneously recorded in the left masseter $(2 \cdot 2 \mathrm{~ms})$. In our experience the temporalis and masseter responses to stimulation at different sites along the trigeminal nerve pathway differ minimally in latency (mean $0.2 \mathrm{~ms}$ ). ${ }^{78}$ In patient 2 , the response in the right masseter was markedly delayed (3.5 $\mathrm{ms}$ on the right, $2 \mathrm{~ms}$ on the left) and dispersed (fig 4).

We concluded that both patients had marked slowing of conduction of the masticatory nerves, more pronounced between the two sites of stimulation (fig 3), without a reduction in amplitude of the $M$ waves evoked by distal stimulation.

Stimulation of the masticatory nerve fibres in both patients also induced autoexcitation with involuntary discharges in the target muscle. In patient 2 , the $M$ wave in the right masseter was often followed by an after-activity consisting of high-frequency $(110 \mathrm{~Hz})$ discharges of synchronised multiple motor unit potentials lasting about $250 \mathrm{~ms}$ (fig 5A). In patient 1 , the $M$ wave in the left temporalis was occasionally followed, after a $10 \mathrm{~ms}$ pause of electrical silence, by a late-activity of asynchronous motor unit potentials, lasting about $30 \mathrm{~ms}$ (fig 5B).

The presence of ephaptic responses in the masseter muscle after stimulation of the deep temporal nerves or in the temporalis muscle after stimulation of the masseteric nerve could not be properly evaluated, because stimulation of the masticatory nerves in the infratemporal fossa is not sufficiently selective. Transcutaneous electrical stimulation of the masseter muscle belly, however, did not evoke ephaptic responses in the temporalis and vice versa, whereas it often triggered prolonged spasms in the affected muscles.
A

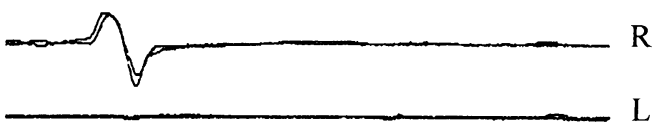

B

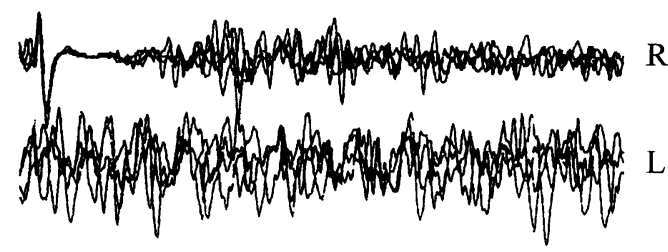

C1

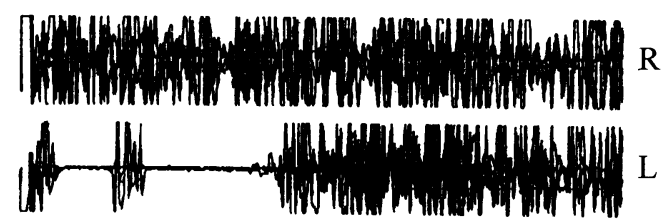

$\mathrm{C} 2$

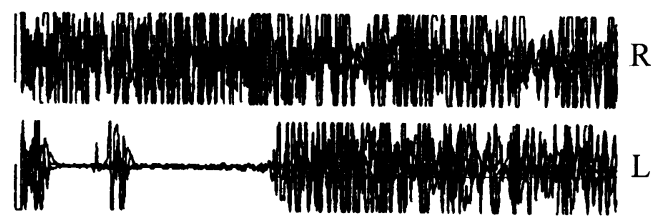

Figure 6 faw jerk and silent periods. The jaw jerk (A) and the silent period $(B)$ evoked by taps to the chin are absent in the left temporalis muscle of patient 1. The early and late silent periods evoked by electrical stimulation of the mentalis nerve $(C)$ are absent in the right masseter of patient 2, after stimulation of the right side (C1) and left side nerve (C2). Calibrations are $5 \mathrm{~ms} / 0.5 \mathrm{mV}$ in $(A)$, and $20 \mathrm{~ms} / 0.5 \mathrm{mV}$ in $(B)$ and $(C)$.

TRIGEMINAL REFLEXES

The jaw jerk evoked by tapping the patient's chin with a triggered hammer ${ }^{1011}$ was recorded through surface electrodes from the masseter and temporalis muscles bilaterally. In patient 1 the jaw jerk was normal and symmetrical in the masseter, whereas it was absent in the left temporalis and normal in the right (right latency $7 \mathrm{~ms}$ ) (fig 6A). In patient 2 the jaw jerk was absent in the right masseter and normal in the left (left latency 7 $\mathrm{ms})$.

The silent period of jaw closing muscles after the chin tap and the early and late silent periods after electrical stimulation of the mental or infraorbital nerve (SP1 and SP2 of the masseter inhibitory reflex, also called exteroceptive suppression) were recorded during maximum clenching of the teeth. ${ }^{1011}$ Although both patients had some difficulty in producing strong and steady contractions without interference by involuntary movements, the silent periods were apparently nor$\mathrm{mal}$ in the unaffected muscles and even in the affected muscles, when these were spasm- 
Table Reports of patients with hemimasticatory spasm demonstrated by EMG examination

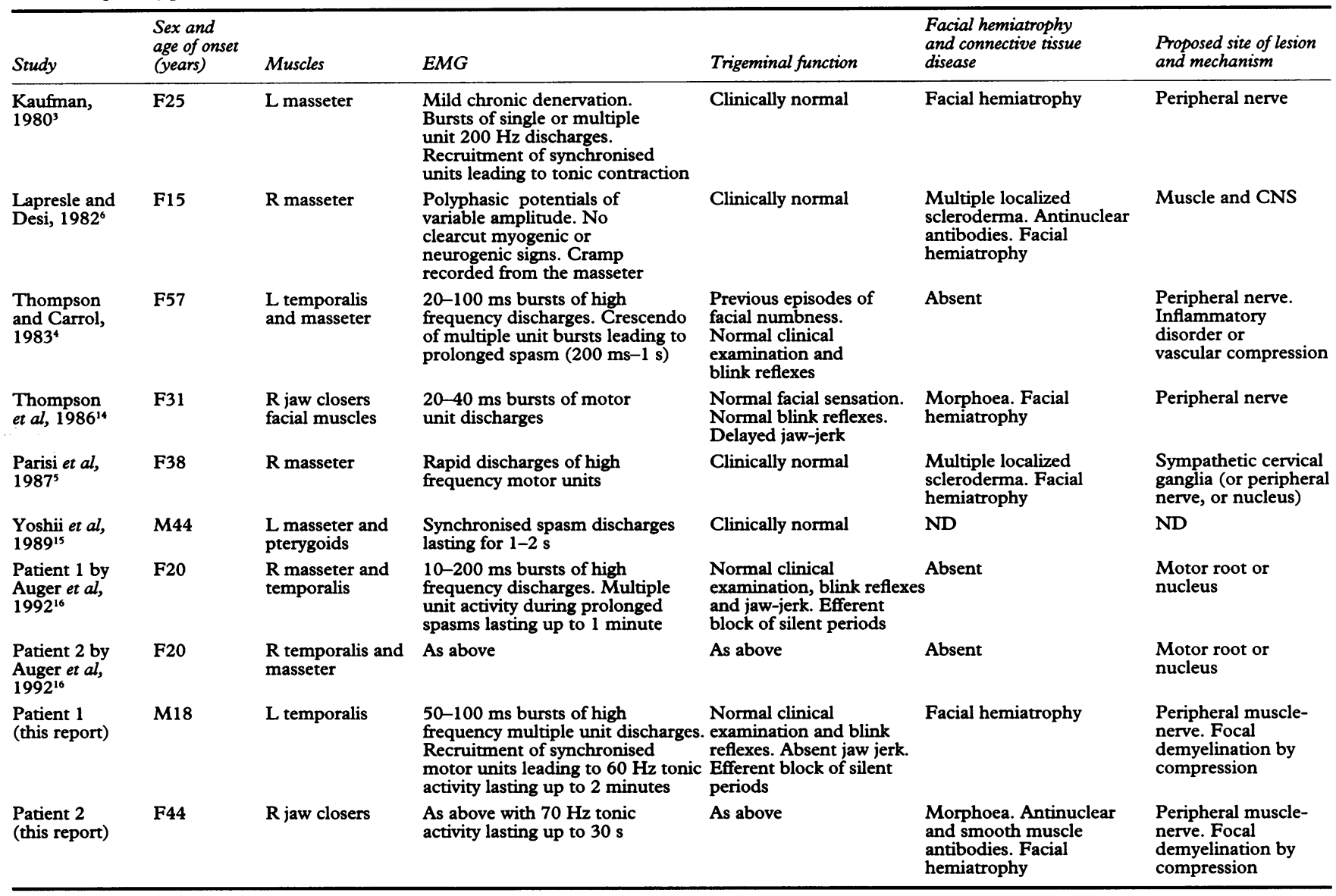

free. During the spasms, the affected muscles-that is, the left temporalis in patient 1 and the right jaw closers in patient 2showed an efferent block: in other words, the silent periods were completely absent in these muscles and normal in the contralateral muscles, regardless of the side of stimulation (fig 6 ). Electrical stimulation of the mentalis and infraorbital nerve on the affected side evoked normal silent periods in the contralateral muscle (patient 1 , right temporalis latency 12 ms, patient 2, left masseter latency $11 \mathrm{~ms}$ ); this finding was important because it allowed us to exclude damage to or interference on the input from cutaneous afferents.

Electrical stimulation of the right and left supraorbital nerves consistently evoked normal and symmetrical early and late blink reflexes in the orbicularis oculi muscles ${ }^{112}$ in both patients.

\section{Discussion}

DIAGNOSIS OF HEMIMASTICATORY SPASM

The occurrence of 'masticatory spasms', in association with facial hemiatrophy, described first in the last century ${ }^{13}$ has since been documented in several clinical reports. The clinical diagnosis is difficult, however, even if the spasm is unilateral, because involuntary movements of the jaw are present in a variety of conditions, including mechanical or inflammatory disorders of the mandible and temporomandibular joint, cephalic tetanus, focal motor epilepsy, tonic spasms of multiple sclerosis, and unilateral dystonia of the jaw. ${ }^{14}$

Clinical ambiguities can easily be resolved by the characteristic electromyographic findings. In this review, we have therefore collected only the reports of unilateral involuntary movements of the jaw corroborated by an EMG description and corresponding to our definition of HMS. As the table shows, these criteria were met in 10 patients only. Two of them are described in the present report, seven were also diagnosed as having unilateral masticatory or masseter spasm, ${ }^{3-5}$ 14-16 $^{-1}$ and one had a different diagnosis. $^{6}$

Hemimasticatory spasm more commonly presents in women $(4: 1)$ in the third and fourth decade (age range 15-57 years). It is often, although not always, associated with facial hemiatrophy. Localised scleroderma and clinical or laboratory signs of connective tissue disease are relatively frequent. Neurological examination is usually normalexcept for the spasm-and facial sensation is always normal. The spasm involves one or more jaw closing muscles on one side, most frequently the masseter. Jaw openers are never affected. Ipsilateral involvement of other cranial muscles has been described in two patients. ${ }^{46}$

Clinically, the involuntary movement consists of brief twitches (resembling those of hemifacial spasm) and prolonged spasms (lasting a few seconds to several minutes, and resembling cramps) or prolonged spasms alone. The spasms are intensely painful, vio- 
lent, and sometimes of sudden onset: during a spasm patients may bite their tongue, ${ }^{14}$ dislocate their temporomandibular joint, ${ }^{3}$ or even break teeth. The involuntary movement may be evoked by yawning, speaking, closing the mouth, chewing, or other voluntary movements of the mouth and jaw, as well as by electrical shocks delivered to the muscle belly or the facial skin.

EMG recordings show no denervation potentials and in most cases the motor unit potentials are normal. The spontaneous activity resembles that of hemifacial spasm (short bursts of $100-200 \mathrm{~Hz}$ discharges of one or few synchronised motor unit potentials), and that of muscle cramps (tonic $50-70 \mathrm{~Hz}$ discharges of a compound potential comprising many synchronised motor unit potentials). The duration of these paroxysms, as well as the number of motor units involved, varies widely from case to case and even from time to time. The hallmarks are high frequency discharges and the recruitment of synchronised motor unit potentials, features that are easily identified and only present (in masticatory muscles) in this rare condition.

The finding of an absent or delayed jaw jerk in the affected muscles is also common, yet not diagnostic, as jaw jerk asymmetries can also be found in multiple sclerosis and even in temporomandibular joint dysfunction. Almost unique to HMS is the finding of an efferent block of the masseter silent periods: during the spasm, no inhibition of the affected muscles can be exerted reflexly by any sensor input, regardless of the site of stimulation. This unusual feature may occur in a few other conditions, one of which is cephalic tetanus. Nevertheless, a complete efferent block to the muscles on one side of the face or even in one muscle alone is exceptional. ${ }^{17}$

To attenuate the spasm, carbamazepine, phenytoin, haloperidol, clonazepam, and diazepam have been tried with varying results, the best being obtained with carbamazepine. Other treatments tried include mandibular anaesthesia, 'rubbing' of the mandibular nerve, ${ }^{3}$ masseter myotomy, ${ }^{14}$ surgical lesions of the motor root, ${ }^{416}$ and local injection with botulinum $\mathrm{A}$ toxin. ${ }^{16}$

\section{PATHOPHYSIOLOGY OF HEMIMASTICATORY SPASM}

We have demonstrated a peripheral motor nerve lesion, as originally proposed. ${ }^{3}$ Nerve conduction studies showed slowing of conduction in the extracranial course of the masticatory nerve fibres, without a reduction in amplitude of the $M$ waves or obvious EMG signs of chronic denervation. Biopsy specimens of the affected temporal muscle appeared histologically normal in both patients. These findings indicate demyelination with sparing of the axon.

The jaw jerk was absent or delayed in several patients (table). This indicates damage to the large diameter ( $\mathrm{A} a$ ) afferent fibres from muscle spindles; a demyelination of even a few of the jaw jerk afferents could easily abol- ish the reflex response. ${ }^{10}$ In contrast, medium size exteroceptive fibres $(A \beta)$ were spared, as shown by the general finding of a normal tactile sensation and normal latency of exteroceptive reflexes when tested (table). Temperature and pain sensation are invariably normal in patients with HMS. One explanation could be that the disease preferentially affects the largest nerve fibres. Alternatively, it only affects muscle nerves, in which the function of small afferent fibres is difficult to assess.

Muscle-nerve damage would explain not only why patients with HMS have no sensory disturbance, but also why they often have spasms in one or two jaw closers only (table), yet never have spasms in the jaw openers, muscles that are innervated by a branch of the inferior dental nerve (fig 3). These observations argue against damage to the motor root or the intracranial portion of the mandibular nerve, where the motor fascicles are closely grouped, ${ }^{18}$ but favour damage to the individual muscle nerves that pass through the infratemporal fossa. This was so in our two patients, in whom the slowing of conduction was prominent in the infratemporal fossa (figs 3 and 4).

The mechanism of facial paroxysmal involuntary activity has been dicussed by Kaufman $^{3}$ and by Thompson and Carroll, ${ }^{4}$ who emphasised the close similarity between hemimasticatory and hemifacial spasm. In our view, our patients and others reported also had cramp-like activity. As in cramps, in HMS the muscle may be hypertrophied, the contractions may also be prolonged, decidedly painful, and sometimes intense enough to produce severe damage to tongue, teeth, and temporomandibular joint. In the EMG, these prolonged spasms nicely fit the description of cramps - that is, irregular motor unit discharges that progressively increase, leading to recruitment of a large part of the muscle and to synchronous discharges at rates from 40 to $60 \mathrm{~Hz}{ }^{12}$ Common to hemifacial spasm and cramps, however, is ectopic excitation. This may be responsible for the high frequency (100-200 Hz) discharges, synchronisation of the whole muscle or more muscles, and after-activity. Synchronisation is tentatively explained by lateral spread of discharges to adjacent nerve fibres, ${ }^{19} 20$ leading to local circuits of re-excitation. After-activity consists of paroxysmal discharges that may follow a voluntary orthodromic contraction or antidromic impulses, ${ }^{21}{ }^{22}$ and is attributed to autoexcitation of the same axons after the passage of an impulse.

In our two patients, we observed the synchronisation of the whole or a large part of the muscle (fig 2C); autoexcitation was demonstrated by recording after-discharges following the direct motor response evoked by stimulation of the masticatory nerves (fig 5). These findings lend support to the hypothesis that the spontaneous activity 'arises' in a demyelinated peripheral nerve. ${ }^{22} 23$ In our patients, unlike those with hemifacial spasm, we were unable to record ephaptic 
responses-that is, 'delayed' responses in one muscle after stimulation of the nerve fibres directed to another muscle. Cross-talking between nerve fibres for different muscles is likely to take place in hemifacial spasm because the supposed site of lesion is at a proximal point, where nerve fascicles are closely grouped. In our patients with HMS, the nerve was damaged extracranially, probably between the infratemporal fossa and the distal nerve branches, where fascicles are more widely separated by perineurial tissue.

\section{ETIOLOGIC CONSIDERATIONS}

Although still debatable, the primary cause of hemifacial spasm is thought to be compression-possibly inducing demyelinationof the nerve near its emergence from the pons. ${ }^{24} 25$ Cramps occur in a number of different conditions associated with neuropathy..$^{2023}$ Evidence has been found of segmental demyelination and axonal degeneration..$^{20}$

The cause of the trigeminal neuropathy that produces masticatory spasms is unknown.

Some patients with HMS also have clinical or laboratory signs of immune connective tissue diseases; these may cause mononeuropathies through several mechanisms. Although connective tissue diseases are often associated with trigeminal damage, these neuropathies nearly always produce sensory impairment alone. ${ }^{27}$ Only three of the 103 patients described by Lecky et $a^{p 8}$ and Hagen et $a l^{29}$ also had signs of a motor trigeminal impairment. In addition, blink reflex as well as histologic studies indicated axonal loss rather than demyelination ${ }^{28}$; in our patients, the absence of motor unit changes in the EMG and the normal muscle biopsy findings ruled out axonal loss.

Motor trigeminal neuropathy is always an exceptional finding, probably arising only when focal pressure causes damage restricted to the muscle nerve. As studies of experimentally induced pressure neuropathies demonstrate, compression can and does lead to ectopic activity. ${ }^{30} 31$

The possibility of cross-compression by an arterial loop-as has been proposed for hemifacial spasm and trigeminal neuralgia-seems unlikely. Despite frequent surgical documentation of cross-compressions between vessels and cranial nerves in the posterior fossa, ${ }^{32} 33$ none of these reports mentions a concurrent motor disturbance resembling masticatory spasm. Furthermore, in two patients with HMS, surgical exploration failed to disclose anomalous vascular contacts along the intracranial $\operatorname{root}^{16}$ or the distal course of the nerve. $^{3}$

In our opinion, when searching for the cause of HMS we should consider its frequent association with facial hemiatrophysurely not a chance event. In the patients reported not to have facial hemiatrophy, this might not be clinically apparent. The process may take 10 years to develop ${ }^{34}$ and muscle spasms are sometimes prodromic to the development of the superficial tissue atrophy. ${ }^{35}$ Facial hemiatrophy often involves not only the skin and subcutaneous tissues, but also deep tissues such as fat, muscles, ligaments, cartilage and bone. ${ }^{34}{ }^{35}$ Deep tissue changes might lead to stretching, angulation, or compression followed by focal demyelination, of the masticatory nerves. Owing to their anatomical relations (fig 3), these nerves may suffer entrapment, in particular the nerves supplying the masseter and temporal muscles, which are most frequently affected in HMS. The temporomasseteric nerve runs in a confined space between the lateral pterygoid muscle and the unyielding surface of the skull; the deep temporal nerves and the masseteric nerve turn sharply around bone crests, and finally run a narrow course between the bone and their own target muscles.

\section{Addendum}

Since this paper was submitted, Auger and colleagues (Neurology 1992;42:2263-6) have reported a third patient with HMS, similar to the two patients described in the table.

1 Gowers WR. A manual of diseases of the nervous system, 2nd ed. Philadelphia: Blakiston, 1897;2:221-4.

2 Wartenberg R. Progressive facial hemiatrophy. Arch Neurol Psychiatry 1945;54:75-96.

3 Kaufman MD. Masticatory spasm in facial hemiatrophy. Ann Neurol 1980;7:585-7.

4 Thompson PD, Carroll WM. Hemimasticatory spasm: a peripheral paroxysmal cranial neuropathy? $\underset{f}{ }$ Neurol Neurosurg Psychiatry 1983;46:274-6.

5 Parisi L, Valente G, Dell'Anna C, Mariorenzi R, et al. A case of facial hemiatrophy associated with linear scleroderma and homolateral masseter spasm. Ital $\mathcal{F}$ Neurol Sci 1987;8:63-5.

6 Lapresle J, Desi M. Sclerodermie avec hemiatrophie faciale progressive et atrophie croisée de l'hèmicorps. Rev Neurol (Paris) 1982;138:815-25.

7 Cruccu G. Intracranial stimulation of the trigeminal nerve in man. I. Direct motor responses. $\mathcal{F}$ Neurol Neurosurg Psychiatry 1986;49:411-8.

8 Cruccu G. Central and peripheral conduction in the human trigeminal motor system, In van Steenberghe D, De Lat A eds. Electromyography of jaw reflexes in man. Leuven: University Press, 1989:3-17.

9 Cruccu G, Berardelli A, Inghilleri $M$, Manfredi $M$. Functional organization of the trigeminal motor system in man. Brain 1989;112:1333-50.

10 Cruccu G, Inghilleri M, Fraioli B, Guidetti B, et al. Neurophysiologic assessment of trigeminal function after surgery for trigeminal neuralgia. Neurology 1987; 37:631-8.

11 Ongerboer de Visser BW. Anatomical and functional organization of reflexes involving the trigeminal system in man: jaw reflex, blink reflex, corneal reflex and exteroceptive suppression. Adv Neurol 1983;39:729-38.

12 Kimura J. Electrodiagnosis in diseases of nerve and muscle: principles and practice, 2nd edn. Philadelphia: FA Davis, 1989.

13 Sachs B. Progressive facial hemiatrophy with some unusual symptoms. Med Rec 1890;37:292-7.

14 Thompson PD, Obeso JA, Delgado G, Marsden CD. Focal dystonia of the jaw and the differential diagnosis of unilateral jaw and masticatory spasm. I Neurol Neurosurg Psychiatry 1986;49:651-6.

15 Yoshii K, Seki Y, Aiba T. A case of unilateral masticatory spasm without hemifacial atrophy. No To Shinkei 1989;41:343-6.

16 Auger RG, Litchy WJ, Ahlskog JE, Cascino TL. Hemimasticatory spasm: clinical and electrophysiologic observations. Neurology 1992;42(suppl 3):352P.

17 Ongerboet de Visser BW, Cruccu G, Manfredi $M$, Koelman JHTM. Effects of brainstem lesions on the masseter inhibitory reflex. Functional mechanisms of reflex pathways. Brain 1990;113:781-92.

8 Pennisi E, Cruccu G, Manfredi M, Palladini G. Histometric study of myelinated fibers in the human trigeminal nerve. F Neurol Sci 1991;105:22-8.

19 Nielsen VK. Pathophysiology of hemifacial spasm: II. Lateral spread of the supraorbital nerve reflex. Neurology 1984;34:427-31 
20 Thompson PD. Stiff people. In Fahn S, Marsden CD, eds. Movement disorders 3. London: Butterworths, 1993 . 367-99.

21 Auger RG. Hemifacial spasm: clinical and electrophysiologic observations. Neurology 1979;29:1261-72.

22 Nielsen VK. Pathophysiology of hemifacial spasm: I Ephaptic transmission and ectopic excitation. Neurology 984;34:418-26.

23 Rowland LP. Cramps, spasm and muscle stiffness. Rev Neurol (Paris) 1985;141:261-73.

24 Auger RG, Piepgras DG, Laws ER Miller RH. Microvascular decompression of the facial nerve for hemifacial spasm: Clinical and electrophysiologic observations. Neurology 1981;31:346-50.

25 Nielsen VK, Jannetta PJ. Pathophysiology of hemifacial spasm: III. Effects of facial nerve decompression Neurology 1984;34:891-7.

26 Welch LK, Appenzeller O, Bicknell JM. Peripheral neuropathy with myokimia, sustained muscular contraction and continuous motor unit activity. Neurology 1972;22: and cont 161 .

27 Hughes RAC. Diseases of the fifth cranial nerve. In Dyck PJ, et al, eds. Peripheral Neuropathy, 3rd edn. Philadelphia: WB Saunders, 1993:801-17.
28 Lecky BR, Hughes RAC, Murray NM. Trigeminal sensory neuropathy: a study of 22 cases. Brain 1987; 110:1463-85.

29 Hagen NA, Stevens JC, Michet CJ Jr. Trigeminal sensory neuropathy associated with connective tissue diseases. Neurology 1990;40:891-6.

30 Burchiel KJ. Abnormal impulse generation in focally demyelinated trigeminal roots. $\mathscr{f}$ Neurosurg 1980;53: 674-83.

31 Rasminsky M. Ectopic impulse generation in pathologic nerve fibers. In Dyck PJ, Thomas PK, Lambert EH, Bunge R, eds. Peripheral neuropathy, vol. 2, 2nd edn. Philadelphia: WB Saunders, 1984:911-8.

32 Jannetta PJ. Microsurgery of cranial nerve cross-compression. Clin Neurosurg 1979;26:607-15.

33 Jannetta PJ. Surgical treatment: Microcascular decompression. In Fromm GH, Sessle BJ, eds. Trigeminal neuralgia. Boston: Butterworth-Heinemann, 1991:145-57.

34 Bruyn GW, Bruyn RPM. Hemiatrophies and hemihypertrophies. In de Jong JMBV, ed. Handbook of clinical neutrophies. In de Jong JMBV, ed. Handbook of clinical
rology, vol. 15. Amsterdam: Elsevier, 1991:475-86.

rology, vol. 15. Amsterdam: Elsevier, 1991:475-86.
35 Delaire J, Lumineau JP, Mercier J, Plenier V. Syndrome de Romberg. Hemi-atrophie faciale progressive. Rev Stomatol Chir Maxillofac 1983;84:313-321.
S 